polar is degenerate; for $p=3, n=3+1, \epsilon=1$, we find again the $2 \mathrm{~d}$ polar is degenerate.

If $n=\alpha p^{m}+\beta p^{m-1}+\cdots+\gamma p^{2}+\delta p$, i.e. $\epsilon=0$ in $n$, then all the polars of $(1,0,0)$ pass through $(1,0,0)$ whether or not this point lies on $f(x, y, z)=0$.

If $n<p$ we find no peculiarities like the above.

Syracuse University

\title{
THE CHARACTERISTIC EQUATION OF A MATRIX*
}

BY E. T. BROWNE

1. Introduction. Consider any square matrix $A$, real or complex, of order $n$. If $I$ is the unit matrix, $A-\lambda I$ is called the characteristic matrix of $A$; the determinant of the characteristic matrix is called the characteristic determinant of $A$; the equation obtained by equating this determinant to zero is called the characteristic equation of $A$; and the roots of this equation are called the characteristic roots of $A$. If $A$ happens to be a matrix of a particular type certain definite statements may be made as to the nature of its characteristic roots. For example, if $A$ is Hermitian its characteristic roots are all real; if $A$ is real and skewsymmetric, its characteristic roots are all pure imaginary or zero; if $A$ is a real orthogonal matrix, its characteristic roots are of modulus unity. However, if $A$ is not a matrix of some special type, no general statement can be made as to the nature of its characteristic roots. In 1900 Bendixson $\dagger$ proved that if $\alpha+i \beta$ is a characteristic root of a real matrix $A$, and if $\rho_{1} \geqq \rho_{2} \geqq \cdots \geqq \rho_{n}$ are the characteristic roots (all real) of the symmetric matrix $\frac{1}{2}\left(A+A^{\prime}\right)$, then $\rho_{1} \geqq \alpha \geqq \rho_{n}$. The extension to the case where the elements of $A$ are com-

\footnotetext{
* Presented to the Society, December 28, 1927.

$\dagger$ Bendixson, Sur les racines d'une équation fondamentale, Acta Mathematica, vol. 25 (1902), pp. 359-365.
} 
plex was made by Hirsch* in 1902. In 1904 Bromwich $\dagger$ further extended the theorem as follows: If $\alpha+i \beta$ is a characteristic root of a matrix $A$ whose elements are real or complex, and if $\rho_{1}, \rho_{2}, \cdots, \rho_{n}$ are the characteristic roots (all real) of $\frac{1}{2}\left(A+\bar{A}^{\prime}\right)$ and $i \mu_{1}, \cdots, i \mu_{n}$ are the characteristic roots of $\frac{1}{2}\left(A-\bar{A}^{\prime}\right)$, then $\alpha$ lies between the greatest and the least of $\rho_{1}, \cdots, \rho_{n}$, and $|\beta|$ does not exceed the greatest of $\left|\mu_{1}\right|, \cdots,\left|\mu_{n}\right|$.

In some cases the theorems just cited give very good limits for the characteristic roots of a matrix, while in other cases the limits are not so restricted. Thus in the case of a real orthogonal matrix these theorems may merely state that the characteristic roots lie in the square $x= \pm 1, y= \pm 1$. In this paper we shall give a criterion which in some cases, notably in the case of a real orthogonal matrix, give more restricted limits than the theorems above.

2. Reduction of a Matrix to a Semi-Unitary Form. Let $A$ be any square matrix of order $n$. Then $A \bar{A}^{\prime}$ is Hermitian and there exists a unitary matrix $\kappa$ (that is, $\kappa \bar{\kappa}^{\prime}=I$ ) such that

$$
\kappa A \bar{A}^{\prime} \bar{\kappa}^{\prime}=M,
$$

where $M \ddagger$ is zero except in the diagonal, and the elements in the diagonal are the (real) characteristic roots $\rho_{1}, \rho_{2}, \cdots, \rho_{n}$ of $A \bar{A}^{\prime}$. We may write

$$
M=\kappa \bar{\kappa}^{\prime} \kappa \bar{A}^{\prime} \bar{\kappa}^{\prime}=B \bar{B}^{\prime},
$$

where

$$
B=\kappa A \bar{\kappa}^{\prime} .
$$

From (1) the elements $b_{i j}$ of $B$ evidently satisfy the conditions

$$
\sum_{t}^{1, \cdots, n} b_{i t} \bar{b}_{j t}=\rho_{i} \delta_{i j}, \quad(i, j=1, \cdots, n),
$$

* Hirsch, Acta Mathematica, vol. 25 (1902), p. 367.

$\dagger$ Bromwich, On the roots of the characteristic equation of a linear substitution, Acta Mathematica, vol. 30 (1906), pp. 295-304.

$\ddagger$ Hilton, Homogeneous Linear Substitutions, Oxford, 1914, p. 41. 
where $\delta_{i j}$ is the Kronecker symbol, and equals 1 if $i=j$; 0 if $i \neq j$. In view of the conditions (3) we shall say that $B$ is in a semi-unitary (semi-orthogonal, if $B$ is real) form. If $\rho_{i}=1,(i=1, \cdots, n), B$ is unitary. We may then state the following theorem.

Theorem I. If $A$ is any square matrix of order $n$ there exists a unitary matrix $\kappa$ such that $\kappa A \bar{\kappa}^{\prime}=B$, where $B$ is in a semi-unitary form.

If $M$ is of rank $r, \kappa$ may be so chosen that $\rho_{i}>0$, $(i=1, \cdots, r) ; \rho_{i}=0,(i=r+1, \cdots, n)$. Since $\rho_{i}=\sum_{t} b_{i t} \bar{b}_{i t}=0$, $(i=r+1, \cdots, n)$, evidently $b_{i t}=0, \quad(i=r+1, \cdots, n ; \quad t=1$, $\cdots, n)$; that is, the last $n-r$ rows of $B$ consist entirely of zeros, so that $B$ is of rank at most $r$. Hence, $B$ must be of rank exactly $r$. Since the rank of $A$ equals the rank of $B$, and the rank of $A \bar{A}^{\prime}$ equals the rank of $M$, incidentally we have given a proof of the following well known theorem.

THEOREM. If $A$ is any square matrix of order $n$, the ranks of $A$ and $A \bar{A}^{\prime}$ are the same.*

3. The Characteristic Roots of $A \bar{A}^{\prime}$. Referring to the matrix $B$ defined as in (1) and (2), let us form a non-singular matrix $C=\left(c_{i j}\right)$ by replacing the zeros in the last $n-r$ rows of $B$ by elements $\left(x_{s 1}, x_{s 2}, \cdots, x_{s n}\right) \neq(0,0, \cdots, 0)$, such that

(4) $\sum_{t}^{1, \cdots, n} b_{i t} \bar{x}_{s t}=0, \quad(i=1, \cdots, r ; s=1, \cdots, n-r)$, and, moreover, such that

$$
\sum_{t}^{1, \cdots, n} x_{i t} \bar{x}_{j t}=0, \quad(i, j=1, \cdots, n-r ; i \neq j) .
$$

Thus, we may find $\left(\bar{x}_{11}, \bar{x}_{12}, \cdots, \bar{x}_{1 n}\right)$ by determining a nonzero solution of the $n-r$ linear homogeneous equations (4). Having obtained $\left(x_{11}, \cdots, x_{1 n}\right)$ we may proceed to find

* Hilton, Homogeneous Linear Substitutions, Exercise 4, p. 51. 
$\left(\bar{x}_{21}, \bar{x}_{22}, \cdots, \bar{x}_{2 n}\right)$ by adjoining to the system (4) the additional linear homogeneous equation

$$
\sum_{t}^{1, \cdots, n} x_{1 t} \bar{x}_{2 t}=0
$$

and so on. If $\sum_{t}^{1, \cdots, n} c_{i t} \bar{c}_{i t}=\rho_{i},(i=1, \cdots, n)$, then $\rho_{i}>0$ and if we write

$$
\chi_{i j}=\frac{c_{i j}}{\left(\rho_{i}\right)^{1 / 2}}, \quad(i, j=1, \cdots, n)
$$

the matrix $\chi$ thus obtained is a unitary matrix. It is evident from the manner in which $\chi$ was built up that $B \bar{\chi}^{\prime}$ is zero except in the diagonal. The elements in the last $n-r$ places in the diagonal are also zero, while those in the first places are $\left(\rho_{i}\right)^{1 / 2}$, the square roots of the characteristic roots of $A \bar{A}^{\prime}$. Since $B \bar{\chi}^{\prime}$ is real and symmetric, the characteristic roots of

$$
N=\chi \bar{B}^{\prime} B \bar{\chi}^{\prime}=\left(B \bar{\chi}^{\prime}\right)^{2}
$$

are the squares of the characteristic roots of $B \bar{\chi}^{\prime}$, and are therefore the characteristic roots of $A \bar{A}^{\prime}$. But

$$
N=\chi \bar{B}^{\prime} B \bar{\chi}^{\prime}=\chi \kappa \bar{A}^{\prime} \bar{\kappa}^{\prime} \kappa A \bar{\kappa}^{\prime} \bar{\chi}^{\prime}=\chi \kappa \bar{A}^{\prime} A \bar{\kappa}^{\prime} \bar{\chi}^{\prime}=\psi \bar{A}^{\prime} A \bar{\psi}^{\prime},
$$

where $\psi$ is the unitary matrix $\chi \kappa$. Thus it follows* that the characteristic roots of $\bar{A}^{\prime} A$ are the same as those of $N$ and therefore of $A \bar{A}^{\prime}$. Hence we have the following theorem.

THEOREM II. If $A$ is any square matrix of order $n$ the characteristic roots of $A \bar{A}^{\prime}$ are the same as the characteristic roots of $\bar{A}^{\prime} A$.

Since the unitary matrices $\kappa, \chi$ above are such that

$$
\kappa A \bar{\kappa}^{\prime}=B \text {, and } B \bar{\chi}^{\prime}=\chi \bar{B}^{\prime},
$$

it follows at once that

Hence

$$
\kappa A \bar{\kappa}^{\prime} \bar{\chi}^{\prime}=B \bar{\chi}^{\prime}=\chi \bar{B}^{\prime}=\chi \kappa \bar{A}^{\prime} \bar{\kappa}^{\prime} .
$$

\footnotetext{
* Hilton, Homogeneous Linear Substitutions, p. 20.
} 


$$
\bar{\kappa}^{\prime} \bar{\chi}^{\prime} \kappa A \bar{\kappa}^{\prime} \bar{\chi}^{\prime} \kappa=\bar{A}^{\prime}
$$

Writing $\bar{\kappa}^{\prime} \bar{\chi}^{\prime} \kappa=\phi$, we have the following theorem.

Theorem III. If $A$ is any square matrix of order $n$ there exists a unitary matrix $\phi$ such that

$$
\phi A \phi=\bar{A}^{\prime} .
$$

In this connection compare Hilton, Homogeneous Linear Substitutions, Ex. 6, p. 124.

Since from (5)

$$
A \phi=\bar{\phi}^{\prime} \bar{A}^{\prime}=(\overline{A \phi})^{\prime},
$$

$A \phi$ is Hermitian, so that we have the following theorem.

THEOREM IV. If $A$ is any square matrix of order $n$, there exists a unitary matrix $\phi$ such that $A \phi$ is Hermitian.

4. The Characteristic Roots of $A$. From (2) the characteristic roots of $A$ are evidently the same as the characteristic roots of $B$. Suppose then that $\lambda$ is a characteristic root of $B$ so that there exists a set $\left(x_{1}, x_{2}, \cdots, x_{n}\right) \neq(0,0, \cdots, 0)$ such that

$$
\sum_{t}^{1, \cdots, n} b_{t i} x_{t}=\lambda x_{i}, \quad(i=1, \cdots, n) .
$$

Taking the conjugates of both members of each of these equations, we have

$$
\sum_{s}^{1, \cdots, n} \bar{b}_{s i} \bar{x}_{s}=\bar{\lambda} \bar{x}_{i}, \quad(i=1, \cdots, n) .
$$

Multiplying corresponding equations in (6) and (7), member for member, and summing as to $i$, we find

that is

$$
\sum_{s, t}^{1, \ldots, n}\left[\sum_{i}^{1, \ldots, n} b_{t i} \bar{b}_{s i}\right] x_{t} \bar{x}_{s}=\lambda \bar{\lambda} \quad \sum_{i}^{1, \cdots, n} x_{i} \bar{x}_{i}
$$

$$
\sum_{i}^{1, \ldots, n} \rho_{i} x_{i} \bar{x}_{i}=\lambda \bar{\lambda} \quad \sum_{i}^{1, \ldots, n} x_{i} \bar{x}_{i} .
$$


Let $G$ be the largest and $s$ the smallest of the characteristic roots of $A \bar{A}^{\prime}$. Then

$$
\lambda \bar{\lambda} \sum x_{i} \bar{x}_{i} \leqq G \sum x_{i} \bar{x}_{i},
$$

so that $\lambda \bar{\lambda} \leqq G$. Similarly, $\lambda \bar{\lambda} \geqq s ;$ i. e.,

$$
s \leqq \lambda \bar{\lambda} \leqq G .
$$

In particular, if $A$ is unitary so that $A \bar{A}^{\prime}=I$, then $G=s=1$, so that $1 \leqq \lambda \bar{\lambda} \leqq 1$; i.e., $\lambda \bar{\lambda}=1$, as is well known. Hence we have the following theorem.

THEOREM V. If $\lambda$ is a characteristic root of a square matrix $A$ and if $G$ and $s$ are respectively the largest and the smallest characteristic roots of $A \bar{A}^{\prime}$, then

$$
s \leqq \lambda \bar{\lambda} \leqq G .
$$

The University of North Carolina 